DOE/ER/13188-8

\title{
NITROGEN CONTROL OF CHLOROPLAST DIFFERENTIATION
}

PEa

Annual Progress Report

JUR $2+\cdots$

$0: \quad$

Dr. Gregory W. Schmidt

University of Georgia Research Foundation, Inc.

Athens, GA 30602

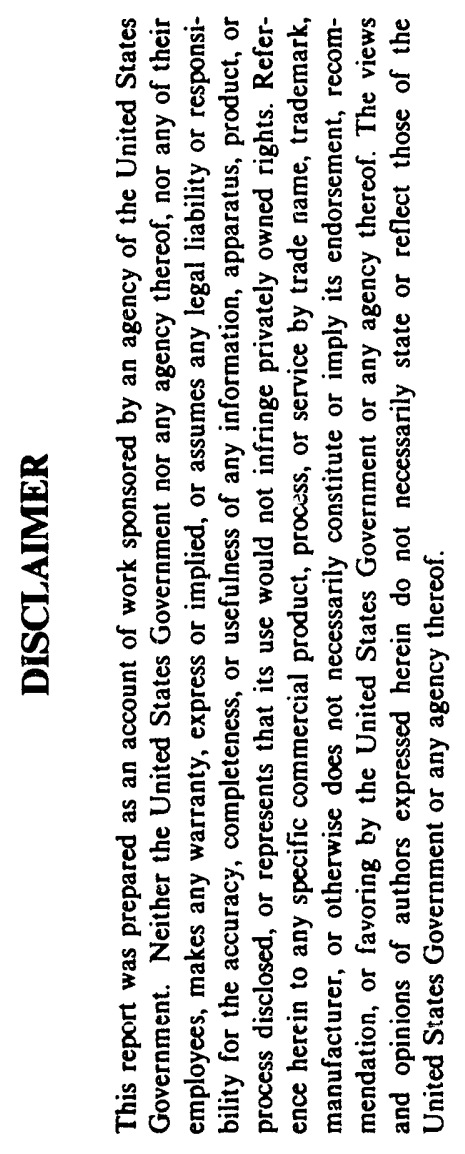

July 1992

PREPARED FOR THE

U.S. DEPARTMENT OF ENERGY

UNDER GRANT

DE-FG09-84ER13188 


\section{Project Abstract}

This project is directed toward understanding how the availability of nitrogen affects the accumulation of chloroplast pigments and proteins that function in energy transduction and carbon metabolism. Molecular and biochemical analyses are performed with Chlamydomonas reinhardtii grown in a continuous culture system: ammonium concentration is maintained at a sufficiently low steady-state concentration that it limits the rate of cell division. As compared to chloroplasts from cells grown with non-limiting nitrogen provisions, chloroplasts of $\mathrm{N}$-limited cells are profoundly chlorophyll-deficient but still assimilate carbon for deposition of large amounts of starch as well as storage lipids. Chlorophyll deficiency arises, in part, by attenuation of the accumulation of nuclear-encoded mRNAs of light-harvesting proteins for both Photosystems I and Photosystem II and, in part, by depressed rates of translation of chloroplast mRNAs for apoproteins of reaction centers. Chloroplast translational effects can be partially ascribed to diminished rates of chlorophyll biosynthesis in N-limited cells, but we have found that pigment levels are not determinants for expression of the nuclear light-harvesting protein genes. Consequently, other signals that are responsive to nitrogen availability mediate transcriptional or posttranscriptional processes for accumulation of the mRNAs for LHC apoproteins and other mRNAs whose abundance is dependent upon high nitrogen levels in the growth medium. Conversely, limited nitrogen availability promotes accumulation of other proteins involved in carbon metabolism and oxidative electron transport in chloroplasts. Hence, thylakoids of N-limited cells exhibit enhanced chlororespiratory activities wherein oxygen serves as the electron acceptor in a pathway that indisputably involves plastoquinone and other electron carrier proteins that remain to be thoroughly characterized. Ongoing and future studies are outlined for 1) further molecular characterization of the identity of nuclear-encoded, nitrogen regulated genes; 2) elucidation of transcriptional vs. posttranscriptional processes that may be involved in LHC mRNA accumulation; 3) identification of the regulatory sequences that serve in the regulation of transcript accumulation in the nuclear compartment; 4) biochemical and molecular analyses of the protein components of chlororespiration. 


\section{Background and Significance}

A large amount of work by many laboratories has been devoted toward understanding the molecular basis for light-dependent chloroplast biogenesis in angiosperms, but little is known about the means by which other environmental determinants also modulate the formation and function of the photosynthetic apparatus. Even though the textbook doctrine for nitrogendeficiency is that it is the most common cause of chlorosis, nothing was known about the molecular basis for this phenomenon. Consequently, we have embarked upon a long-term project to discern how chloroplast structure and function is modified when this nutrient is provided at levels that are sufficiently low to restrict cell growth. A simplistic hypothesis might be put forth that nitrogen-deficiency symptoms would arise merely because it is a necessary constituent of nucleotides, amino acids and chlorophylls, but it should be recognized that nitrogen assimilation products have been identified as key entities in the regulation of gene expression in prokaryotes and lower eukaryotes (Arst et al., 1990; Atkinson and Fisher, 1991; Caddick and Arst, 1990; Cunningham and Cooper, 1991; Fu and Marzluf, 1990; Hoover et al., 1990; Kudla et al., 1990; Macaluso et al., 1990; Martin-Nieto et al., 1991; Minehart and Magasanik, 1991; Young et al., 1990). Moreover, nitrogen-deficiency is a condition for the transition into sexual reproductive cycles as well as such gross alterations of grow h form in yeast where cells can become filamentous (Gimeno et al. 1992). From these examples, nitrogen should be perceived as a forceful regulatory agent in eukaryotes, but one that has been largely ignored in studies of photosynthetic gene expression in plants and algae. A more thorough understanding of the regulatory effects of nitrogen and other nutrients is necessary for a comprehensive understanding of how environmental signals mediate the growth and development of photosynthetic organisms.

The model system for our studies of nitrogen-dependent regulation of chloroplast differentiation is the unicellular green alga Chlamydomonas reinhardtii which is grown phototrophically in a continuous culture system. When $150 \mu \mathrm{M}$ nitrogen is provided at a dilution rate of 0.25 volumes of the growth medium per day, the cultures are sustained at a density of less than $10^{5}$ cells $/ \mathrm{ml}$ and chlorophyll deficiency becomes quite pronounced. We previously found (Plumley and Schmidt, 1989; Plumley et al., 1989) that there is a concomitant loss of lightharvesting complexes and reduced levels of Photosystem II reaction center complexes while ATP synthetase and Photosystem I reaction centers are maintained at high levels. Moreover, it was shown that reduced rates of chlo:oplast protein synthesis are due to differential effects on mRNA translation. In contrast, the deficiency of light-harvesting genes is due to marked reductions of the nuclear-encoded $c a b$ mRNAs (Plumley and Schmidt, 1989). Although there is no significant reduction of the amounts of RuBPCase, we also detected substantial changes in the mRNA abundance of the alga's two small subunit genes. All of the effects of nitrogen-limitation are readily reversible: greening of cells is completed within 24 hours after provision of $10 \mathrm{mM}$ ammonium. During this time, the plastid translational constraints are disengaged and progressive changes in the abundance of nuclear transcripts occur, including a transient 30 -fold elevation of $c a b$ mRNAs (Plumley and Schmidt, 198؟).

The proposal for the current project concerned three general goals: 1) Studies of photosynthetic gene expression in response to nitrogen deficiency; 2) Further characterization nuclear genes, including those encoding light-harvesting apoproteins, that are differentially expressed in response to nitrogen availability; 3) Further analyses of the components of the oxidative, "chlororespiratory" electron transport activity associated with thylakoids. For this goal, we had obtained preliminary evidence that this activity is highly augmented in nitrogen deficient 
cells. Manuscripts pertaining to goals 1 and 3 are provided as appendices.

\section{Progress During the Current Funding Period (1990-1993)}

\section{Photosynthetic Gene Expression}

We reported that one of the characteristics of nitrogen-limited cells is that chloroplastencoded mRNAs corresponding to $p s b A, p s b B, p s b C, p s b D$, $p s a A 1$, and $p s a A 2$ are maintained at high levels (Plumley and Schmidt, 1989; Plumley et al., 1989). However, quantitative immunological assays showed that the 47 and $43 \mathrm{kDa}$ pigment binding proteins corresponding to $p s b B$ and $p s b C$, respectively, are substantially reduced in abundance while ATP synthetase polypeptides and the Photosystem I reaction center polypeptides from psaA1 and psaA2 accumulate to normal levels when analyzed on a per cell basis. From in vivo pulse-labelling analyses we also discerned that, except for the D-1 polypeptides synthesized from $p s b A$ mRNA, there is a nearly complete absence of synthesis of Photosystem II proteins in N-limited cells. In parallel studies of light-harvesting proteins, we showed that LHCP II polypeptides are not synthesized in N-limited cells because the $c a b$ mRNAs, which usually are quite conspicuous, do not accumulate. From this work, it appeared that $\mathrm{N}$-limitation is accompanied by differential regulatory processes exerted at the translational level within plastids. However, it remained unclear whether this is due to a direct effect of $\mathrm{N}$-limitation or an indirect effect due to reduced rates of chlorophyll synthesis. Work from other laboratories had indicated that chlorophyll synthesis is required for translation of chloroplast mRNAs encoding chlorophyll-binding proteins (Gamble, et al., 1988; Klein and Mullet, 1986, 1987; Klein et al., 1988; Kreuz et al., 1986; Laing et al., 1988; Mullet, 1988) but subsequent studies led to the conclusion that the reaction center apoproteins are degraded when chlorophyll assembly is impaired (Eichacher et al., 1990; Mullet et al., 1990).

The coordination of pigment synthesis with the accumulation of the chlorophyll-binding apoproteins synthesized within chloroplasts is of obvious relevance as a potential aspect of the regulation of chloroplast biogenesis by nitrogen. Thus, we wished to address issues of how pigment biosynthesis affects chloroplast as well as nuclear gene expression in Chlamydomonas. As shown in Appendix I (Herrin et al., 1992), this was achieved by studies of mutants that possess either conditional or constitutive defects in chlorophyll and/or carotenoid biosynthesis.

Importantly, with carotenoid mutants that accumulate either phytoene or $\zeta$-carotene, normal rates of chlorophyll $a$ and $b$ synthesis can be discerned in autoradiographs of thin-layer chromatographs but only a small portion $(\sim 10 \%)$ of these pigments survive subsequent in vivo degradation. Although the chlorophyll deficiency of carotenoid biosynthesis mutants or plants treated with inhibitors of carotenoid formation has long been appreciated, this is the first report that carotenoid affects accumulation, rather than synthesis of chlorophylls $a$ and $b$. However, the carotenoid effect is probably due to impaired assembly of the chlorophylls with reaction center and light-harvesting apoproteins as opposed to a direct mediation of chlorophyll stabilization. This conjecture follows from immunological demonstrations that pigment-binding proteins are not detected in thylakoids from the carotenoid (and chlorophyll) synthesis mutants (Appendix I). Moreover, pulse- and pulse/chase-labelling studies showed that there is detectable synthesis of light-harvesting and reaction center apoproteins, but usually at reduced rates, in both the chlorophyll and carotenoid mutants. Except for the occurrence of low levels of $c a b$ mRNA in 
dark-giown strains that require light for chlorophyll synthesis, we found that there are no effects of either carotenoid or chlorophyll synthesis on the levels of apoprotein mRNAs of either nuclear or chloroplast origin. Consequently, we concluded that assembly of both pigment classes is required for stabilization of newly synthesized apoproteins and that the deficiencies of reaction center and light-harvesting proteins in the mutant thylakoids is primarily due to posttranslational degradation. Moreover, we deduced that carotenoid and chlorophyll synthesis also exert a partial effect on the translation of chloroplast and cytoplasmic mRNAs for pigment-binding proteins because of their relatively low rates of synthesis. Finally, in vivo RNA pulse-labelling revealed that $c a b$ transcripts of nuclear origin are subject to posttranscriptional regulation in dark-grown strains requiring light for chlorophyll synthesis.

Although chlorophyll synthesis is required for the efficient translation of chloroplast mRNAs for reaction center proteins and, in a more absolute fashion, the stabilization of newlysynthesized apoproteins, the effects of nitrogen-deficiency on chloroplast synthesis of the thylakoid proteins can be only partly attributed to insufficient chlorophyll synthesis. For example, low chlorophyll abundance does not correlate with the relatively high rates of synthesis of Photosystem I apoproteins in N-limited chloroplasts. Moreover, low rates of chlorophyll synthesis cannot cause the paucity of $c a b$ transcripts in N-limited cells. It is possible that an chlorophyll biosynthesis intermediate between protoporphyrin IX and chlorophyll(ide) may influence stabilization of $c a b$ transcripts (Appendix 1; Herrin et al., 1992), but it would be surprising if such porphyrins would be especially prevalent in $\mathrm{N}$-limited cells. Consequently, we favor the notion that nitrogen availability affects both chloroplast and nuclear gene expression in a manner that is not mediated by pigment intermediates per se.

\section{Differential Nuclear Gene Expression}

From one- and two-dimensional gel electrophoresis analyses of proteins synthesized during pulse-limiting or in vitro translation of mRNAs from N-limited vs. control cultures, it is apparent that there are considerable differences in the identities of the most abundant soluble and membrane proteins synthesized in cells subjected to the two culture conditions (Plumley and Schmidt, 1989; Plumley et al., 1989). Moreover, direct comparisons to cells subjected to heatshock demonstrated that the phenotype of $\mathrm{N}$-limited cells does not correspond to a generalized stress response (Plumley et al., 1989). Finally, cells in N-limited continuous cultures have bypassed differentiation into gametes as evidenced by: 1) sustained division of cells; 2) absence of the morphological features of gametes; 3 ) inability of $\mathrm{N}$-limited cells to undergo mating with authentic gametes of the opposite mating type; 4) absence of gamete-specific mRNAs in N-limited cells as determined via RNA blots with clones provided by Dr. C. Beck (Beck and Acker, 1992). For these reasons and because of the pervasive changes of physiology of N-limited cells, the concept that nitrogen is directly involved in the regulation of differential gene expression becomes compelling. Consequently, we proposed to obtain a collection of nuclear gene clones corresponding to genes that are preferentially expressed in either $\mathrm{N}$-limited or control growth conditions. These would be used not only for identification of proteins that are differentially synthesized in response to nitrogen availability, but also would be necessary for elucidation of regulatory sequences that function in the nitrogen control process.

The strategy for isolation of differentially expressed genes has involved exploitation of the relative small nuclear genome size $\left(1.2 \times 10^{8}\right.$ b.p.) of Chlamydomonas to enable direct differential screening of a lambda genomic library directly with radiolabeled, single-stranded 
cDNAs synthesized from RNA from N-limited and control cells. . With genomic DNA inserts of an average size of $16 \mathrm{~kb}$ and use of plates containing 600 plaques, a genomic equivalent is represented in only 13 plates, but 26 plates were used per screening. One important technical prerequisite for this approach was to optimize reverse transcription conditions such that fulllength cDNAs, primed with oligo-dT and labelled with ${ }^{32} \mathrm{P}$-deoxyribonucleosides, are reproducibly obtained. From four such screening undertakings to date, we picked approximately 250 clones harboring genes that are preferentially expressed in cells grown in control culture conditions and approximately 300 clones that appear to contain genes that are abundantly expressed in $\mathrm{N}$-limited cells. Of these, nearly 300 isolates have have been se'ected for subcloning and for studies of changes in mRNA abundance. At present, we have focussed on 8 clones that contain genes that are control-specific and 10 containing genes expressed predominantly in $\mathrm{N}$-limited cells. The protocol for selection of the appropriate subclones within the large genomic fragments has entailed probing southern blots of restriction digests with ${ }^{32} \mathrm{P}-\mathrm{cDNAs}$ followed by generation of recombinant plasmids (pBlueScript) with excised DNA bands from replicate agarose gels. Secondly, the subclones are employed as probes in Northern blot analyses of RNA purified from control and $\mathrm{N}$-limited cells. In this manner, sibling clones are culled and the extent to which mRNAs levels are differentially affected by the culture conditions can be evaluated. Finally, DNA sequencing has been initiated for those subclones which, on the basis of restriction mapping studies, has enabled unambiguous identification of fragments whose sequences are present in mature RNAs.

Not surprisingly (and serving to validate the screening strategy), one of the genes that is preferentially expressed in response to nitrogen turns out to be $r b c S 2$. This RuBPCase small subunit allele gives rise to the single $0.85 \mathrm{~kb}$ small subunit mRNA found in control cells (Plumley and Schmidt, 1989) but also is preferentially expressed in dark-grown Chlamydomonas cells cultivated with acetate as a carbon growth source (Goldschmidt-Clermont, 1986; GoldschmidtClermont and Rahire, 1986). In response to ammonium, $\mathrm{N}$-limited cells accumulate relatively large amounts of the $1.1 \mathrm{~kb}$ transcript of $r b c S 1$. As yet, sequencing of other clones has not enabled identification of gene products perhaps because the plasmid inserts contain large intron or noncoding sequences. Although more extensive sequence information is forthcoming, this hindrance illustrates one of the predicted short-comings of the gene isolation scheme that might be averted if cDNA libraries instead of genomic libraries were employed: many strongly regulated genes have not been identified because they are present on large fragments that also contain genes that are strongly expressed in a constitutive manner. Also, those differentially expressed genes that give rise to low abundance mRNAs wou!d be difficult to discern upon screening of either genomic or cDNA libraries since, in the latter case, the number of cDNA clones would be relatively low unless procedures that select against the reverse transcriptase products of highly abundant mRNAs are utilized.

Another approach for elucidation of differential gene expression in response to nitrogen availability has been to utilize clones encoding LHC apoproteins and other proteins that are reasoned to be $\mathrm{N}$-regulated. One outcome is that one of the major light-harvesting proteins of 22 $\mathrm{kDa}$ for Photosystem I has been found to be as strongly regulated at the level of mRNA abundance as the genes encoding chlorophyll $a / b$-binding proteins of Photosystem II (Bruns and Schmidt, unpublished). This finding correlates with large changes in the spectroscopic properties of PS I particles, especially with respect to fluorescence excitation and emission spectra (Plumley et al., 1989). 


\section{Chlororespiration}

The photosynthetic properties of $\mathrm{N}$-limited cells, encompassing both carbon metabolism and electron transport, are quite different from that of cells cultured in normal growth media. For this reason, we were inspired to discern the extent to which the enigmatic "chlororespiratory" electron transport pathway is also present in the chlorotic, but starch-laden, cells.

Chlororespiration was first observed by Kok et al. (1949) who unexpectedly found that there is not a linear effect of increasing light intensity on oxygen uptake by Chlorella. He concluded that, in addition to driving photosynthetic oxygen evolution, light must rather cause a "suppression" of oxygen consumption. Healey and Myers (1971) studied this phenomenon further and concluded that the "Kok effect" is due to diversion of reductant away from respiratory electron transport and into Photosystem I because the phenomenon became exaggerated by treatment of Chlamydomonas cells with DCMU. Subsequently, Bennoun $(1982,1983)$ studied the redox state of the thylakoid plastoquinone pool in mutants lacking Photosystem I reaction centers and the cytochrome $b_{6} / f$ complex and, from this work, established the existence of a chlororespiratory pathway. Although the biochemical identities of the electron carriers for chlororespiration remain obscure, chlororespiration must involve plastoquinone and, apparently, as many as 5 electron carriers: a terminal oxidase, a cytochrome b/c complex, a cytochrome c-like moiety, a NAD(P)H dehydrogenase and, possibly, a succinate dehydrogenase.

From Photosystem II fluorescence induction measurements of Chlamydomonas mutants lacking Photosystem I, Bennoun (1982) found that plastoquinol oxidation can occur via a lightindependent pathway and that oxygen serves as the terminal electron acceptor (Bennoun, 1982). Like mitochondrial cytochrome oxidases, the putative terminal complex of thylakoids was found to be sensitive to carbon monoxide, cyanide, and azide but not salicylhydroxamic acid (SHAM), an inhibitor of the alternative oxidase of plant and algal mitochondria (Bennoun, 1982; Peltier and Sarrey, 1988). More direct evidence that a terminal oxidase must be localized in thylakoids was provided by Peltier et al., (1987): inhibition of oxygen uptake by a single light flash of $2 \mu$ second duration was detected in intact wild-type cells of Chlamydomonas deposited onto a bare platinum electrode. No light-inhibition was observed in a mutant lacking Photosystem I, indicating that the effect of illumination is to divert electron flow from plastoquinol to the oxidase and toward Photosystem I. Photosystem II plays no role in the flash-induced signals since DCMU does not change the magnitude of light inhibition of oxygen uptake (Peltier et al., 1987). Moreover, mass spectroscopy analyses of cells exposed to ${ }^{18} \mathrm{O}_{2}$ showed that there is no corresponding effect on $\mathrm{H}_{2}{ }^{16} \mathrm{O}$ photolysis (Peltier et al., 1987). Finally, estimates of the $\mathrm{K}_{\mathrm{M}}$ s for the chloroplast oxidase $(23 \mu \mathrm{M})$ vs. that of the mitochondrial cytochrome oxidase $(0.2 \mu \mathrm{M})$ and the cyanide-insensitive alternative mitochondrial oxidase $(5.5 \mu \mathrm{M})$ established that substrate affinity is another distinguishing property of the three complexes (Peltier et al., 1987). It should be noted that Garab et al. (1989) determined that a cyanide- but also SHAM-sensitive oxidase is present in chloroplasts of tobacco protoplasts and pea seedlings which also functions to oxidize plastoquinol. However, this activity was lost upon thylakoid purification. This is the only convincing report of chlororespiration in vascular plants.

In addition to the terminal oxidase, the chlororespiratory pathway also appcars to involve a cytochrome b/c complex different from that of the photosynthetic cytochrome $b_{6} / f$ complex. Thus, Bennoun (1983) found that a Chlamydomonas mutant that lacks cytochrome $f$ exhibits sustained reduction and oxidation of plastoquinone in the dark, provided the cells are kept 
aerobic. More recently, Ravenel and Peltier (1991) found that myxothiazol and antimycin A, inhibitors of the mitochondrial cytochrome b/c complex (Bennoun et al., 1991), also inhibit chlororespiration but at 10- to 20-times higher concentrations than are necessary to block mitochondrial electron transport. Photosynthetic electron transport is unaffected by these inhibitors. Consequently, it is apparent that Chlamydomonas thylakoids contain an additional cytochrome $b / c$ complex whose inhibitor-sensitivity properties can be distinguished from those of the mitochondrial counterpart and the cytochrome $b_{6} / f$ complex that is specific for photosynthetic electron transport.

Another component of the chlororespiratory electron transport chain is expected to be functionally equivalent to cytochrome $\mathrm{c}$ or plastocyanin, mediating electron flow from the cytochrome $\mathrm{b} / \mathrm{c}$ complex to the terminal oxidase. The only information concerning the identity of such a protein was provided by Bennoun (1983) who found that chlororespiratory activity (dark oxidation of plastoquinol) is unaffected in a Chlamydomonas mutant that lacks plastocyanin. Like other green algae, Chlamydomonas can synthesize a plastocyanin substitute, cytochrome $\mathrm{c}_{6}$ (cytochrome $\mathrm{c}_{552}$ ), especially when cells are grown in copper-deficient medium (cf. Merchant et al., 1991). Merchant et al. (1991) have provided evidence that the cyt $\mathrm{c}_{6}$ gene is tightly controlled by a copper-dependent repressor of transcription. However, transcription of the cyt $\mathrm{c}_{6}$ gene occurs when cells are grown to stationary phase in conventional, copper-containing growth medium. Therefore, it is possible that the cytochrome $\mathrm{c}_{6}$ can have dual functions in photosynthetic and chlororespiratory electron transport. The hypothesis is consistent with the finding that cyanobacterial cytochrome $c_{6}$ is an electron donor to both cytochrome oxidase and Photosystem I (Moser et al., 1991).

Bennoun (1982) showed that either NADH or NADPH could be provided to thylakoids to promote dark reduction of plastoquinone. This evidence for the existence of a functional thylakoid $\mathrm{NAD}(\mathrm{P}) \mathrm{H}$ dehydrogenase complemented the earlier work of Godde and Trebst (1980) who showed that NADH could serve as an electron donor for the Photosystem I-driven Mehler reaction and that the responsible thylakoid NADH dehydrogenase is rotenone-sensitive. Subsequently, partial purification and characterization of the dehydrogenase from Chlamydomonas thylakoids solubilized with Triton X-100 was accomplished by Godde (1982). Gfeller and Gibbs $(1985 \mathrm{a}, \mathrm{b})$ deduced that a rotenone-sensitive thylakoid $\mathrm{NAD}(\mathrm{P}) \mathrm{H}$ dehydrogenase serves as a sink for reducing equivalents generated during starch breakdown and glycolysis: inhibition of plastoquinol oxidation (and $\mathrm{NAD}(\mathrm{P}) \mathrm{H}$ oxidation) by $\mathrm{DBMIB}$ and anaerobic conditions lead to the accumulation of ethanol from acetaldehyde via a chloroplast-localized fermentative pathway.

With the discoveries of chloroplast genome sequences of plant chloroplasts encoding for proteins with similarities to mitochondrial NADH dehydrogenases, a widespread interest has developed in the possible functions of the putative subunits and the extent to which chlororespiration might be a ubiquitous, although a conditional feature of photosynthetic membranes (Ohyama et al., 1986; Shinozaki et al., 1986. Matsubayashi et al., 1987; Hiratsuka et al., 1989; Schantz and Bogorad, 1988; dePamphilis and Palmer, 1990; Sherer, 1990). Although the chloroplast $n d h$ genes are transcribed (Schantz and Bogorad, 1988; Hiratsuka et al., 1989), the presumed protein products have not been detected. Interestingly, at least one achlorophyllous plant has been found to contain plastid genomes from which the $n d h$ genes, along with most of the genes encoding photosynthetic proteins, have been deleted (dePamphillis and Palmer, 1990). This finding implies that the $n d h$ gene products may perform functions that are dependent upon photosynthetic activities or the formation of thylakoids. 
The final complex for consideration as a chlororespiratory constituent is succinate dehydrogenase. With purified chloroplasts from Chlamydomonas, Willeford et al. (1989) found that this enzyme was thylakoid-bound and could serve for the donation of electrons to Photosystem I in a Mehler reaction carried out in the presence of cyanide. They also partially characterized the chloroplast and mitochondrial succinate dehydrogenases and found that they exhibit equivalent $\mathrm{K}_{\mathrm{M}}$ s. Interestingly, of the total cell activity, $30 \%$ of the enzyme appears to be chloroplast localized. Its proposed function is to facilitate oxidation of substrates of the glyoxylate cycle. Assuming that the thylakoid enzyme functions via plastoquinone, it is likely that electron flow to the terminal oxidase could occur as well as light-dependent transfer to Photosystem I.

As documented in Appendix 2 (Peltier and Schmidt, 1991), we discovered that oxidative electron transport activities that are thylakoid-localized are 10 -fold higher in N-limited than in control cells. Correspondingly, $\mathrm{N}$-limited cells exhibit a total respiratory rate that can be 2-fold higher than normal and, from measurements of the light-inhibition of $\mathrm{O}_{2}$ consumption, chlororespiration accounts for $30 \%$ of the additional respiratory capacity. In this study, we employed not only direct measurements of oxygen exchange in flash-illuminated cells but also fluorescence assays to monitor the redox state of the plastoquinone pool. The plastoquinone pool is sustained in a highly reduced state (150\% greater than normal) in $\mathrm{N}$-limited cells even when they are kept in darkness. Accordingly, thylakoids are perpetually in low fluorescent state- 2 which is diagnostic of dissociation of light-harvesting complexes from Photosystem II. Thus, the reduced plastoquinone pool activates thylakoid protein kinases that phosphorylate light-harvesting polypeptides, the core antenna complexes and reaction center polypeptides of Photosystem II (Ikeuchi et al., 1987a,b). Like controls adapted to darkness, illumination of N-limited cells subjected to anaerobic conditions (to arrest chlororespiratory electron flow) undergo a transition to high fluorescence state 1 when plastoquinone reduction is further inhibited by DCMU inhibition of Photosystem II. Interestingly, the rate of this transition is much faster in N-limited cells as compared to control cells. Perhaps this is due to the occurrence of novel light-harvesting components that are presumed to be products of those members of the $c a b$ gene that appear to be uniquely expressed in N-limited cells (see above; Plumley and Schmidt, 1989).

The $\mathrm{K}_{\mathrm{M}}$ for $\mathrm{O}_{2}$ in chlororespiration is 100 -fold higher than that of respiration (Peltier et al., 1987), when the specific activity of chlororespiration increases 10 -fold in N-limited cells, it should be expected that there are large increases in the proteins of the terminal oxidase. We reported that thylakoids of $\mathrm{N}$-limited cells accumulate much higher levels of NAD(P) dehydrogenase and two cytochromes (h1 and h2) that normally are only minor constituents (Peltier and Schmidt, 1991). The NAD(P)H dehydrogenase was isolated from non-denaturing gels and was found to contain two subunits with molecular weights similar to that of the NADH dehydrogenases of cyanobacteria. As the abundance of these proteins correlated with augmented chlororespiratory activities, we supposed that they are components of the oxidative electron transport chain as did Lemaire et al. (1986) for h1 and h2 in their detailed studies of the cytochromes of Chlamydomonas chloroplasts.

In terms of the functional and/or adaptive significance of the augmentation of chlororespiration in $\mathrm{N}$-limited cells, we proposed that the pathway may serve as a protectant from photodamage (Peltier and Schmidt, 1991). Thus, N-limited cells slowly progress through the cell cycle and yet retain the ability fix to carbon unabatedly. Chlororespiration provides a means for carbohydrate and NADPH oxidation. Importantly, photodamage resulti ig from the absence of ample levels of NADP + would be averted. Finally, based on the demonstration that 
chlororespiratory electron flow is coupled with proton translocation (Bennoun, 1983), ATP synthesis as well as the integrity of thylakoids would be sustained. It should be noted that chlororespiration provides a pathway for cyclic electron flow which, at least in Chlamdyomonas, has been observed in cells that have been subjected to photoinhibitory irradiance treatments (Canaani et al., 1989). It is possible that ATP synthesis via a coupling of chlororespiratory and Photosystem I activities could provide the energy needed for protein synthesis that is required for recovery from photodamage.

Recently, the conjecture that cytochromes $h_{1}$ and $h_{2}$ are thylakoid constituents has been severely attacked. Although Chlamydomonas mitochondria have never been purified for biochemical characterization, Atteia et al. (1992) have provided compelling evidence that thylakoid preparations from the alga are contaminated to some extent by inner mitochondrial membranes. Thus, they report that cytochrome $h_{1}$ is absent from thylakoids of a mutant with a deletion of the mitochondrial $c o b$ gene. Atteia et al. (1992) point out that cytochrome $\mathrm{h}_{1}$ could be either (mitochondrial-encoded) cytochrome $b$ or (nuclear-encoded) cytochrome $c_{1}$ since each of the proteins would probably be required for stable accumulation in mitochondria of the remaining cytochrome $b / c$ subunits. Moreover, they deduce from immunological and protein sequence analyses that the $h_{2}$ cytochrome is soluble cytochrome $c$, but membrane encapsulated, and that the mitochondrial B subunit of ATP synthase is a relatively minor protein recovered with thylakoids.

It remains possible that cytochromes $h_{1}$ (if it corresponds to cytochrome $c_{1}$ ) and $h_{2}$ could be imported to both mitochondria or chloroplasts (Bennoun, 1981; Hurt et al., 1986), especially in cells subjected to $\mathrm{N}$-limitation when augmented synthesis of the precursors would most likely occur. We noted that neither the mitochondrial transcripts from the coxI or nad5 (urf5) loci are up-regulated in N-limited cells (Plumley and Schmidt, 1989). Moreover, the abundance of mitochondrial DNA (and, presumably, mitochondrial size and number) are not affected by Nlimited culture conditions (Plumley and Schmidt, 1989). However, work in our laboratory also has led to the keen appreciation that contamination of thylakoid preparations with mitochondrial membranes confounds identification of the true chlororespiratory complexes. Consequently, Jeff Blanchard (a second year graduate student) has been devising methods for monitoring the extent of contamination and developing protocols for obtaining $\mathrm{N}$-limited thylakoids and mitochondrial membranes in a highly purified state. Purification of intact chloroplasts (Mason et al., 1991) from Chlarnydomonas affords little improvement in the removal of mitochondria since adherence of these and other organelles to chloroplast envelopes and to remnants of the interconnecting cytoskeleton can he readily perceived in electron micrographs. Thus, we have continued using thylakoids enriched by flotation during sucrose step gradient centrifugation followed by differential centrifugation aided by $\mathrm{Mg}++$ titration: mitochondrial inner membranes are likely to have a lower negative surface charge relative to that of thylakoid: (Ericson, 1974; Barber and Chow, 1979). Alternatively, polyclonal antibodies that react vith exposed regions of residual light-harvesting proteins can be utilized but this method becomes impractical for large-scale preparations. Mitochondrial inner membrane contamination is monitored by measurements of the presence of cardiolipin by two-dimensional thin-layer chromatography. With these techniques, it has been possible to obtain thylakoid preparations that still are enriched in cytochromes $h_{1}$ and $h_{2}$ and which also contain rotenone-sensitive NADH dehydrogenase. Fractions depleted of cytochromes $h_{1}$ and $h_{2}$, but still containing substantial amounts of thylakoid proteins, possess only a rotenone-insensitive $\mathrm{NADH}$-dehydrogenase, presumably the aiiernative ubiquinone oxidoreductase of mitochondrial origin. Unfortunately, cardiolipin is present in all membrane 
preparations obtained so far. Consequently, we are embarking on the use of the twophase polymer partitioning system modeled on that described by Larson (1983). The goals of these efforts are not only to establish the identities of chlororespiratory complexes of $\mathrm{N}$-limited thylakoids but also to characterize more fully the proteins of mitochondrial membranes from Chlamydomonas. 

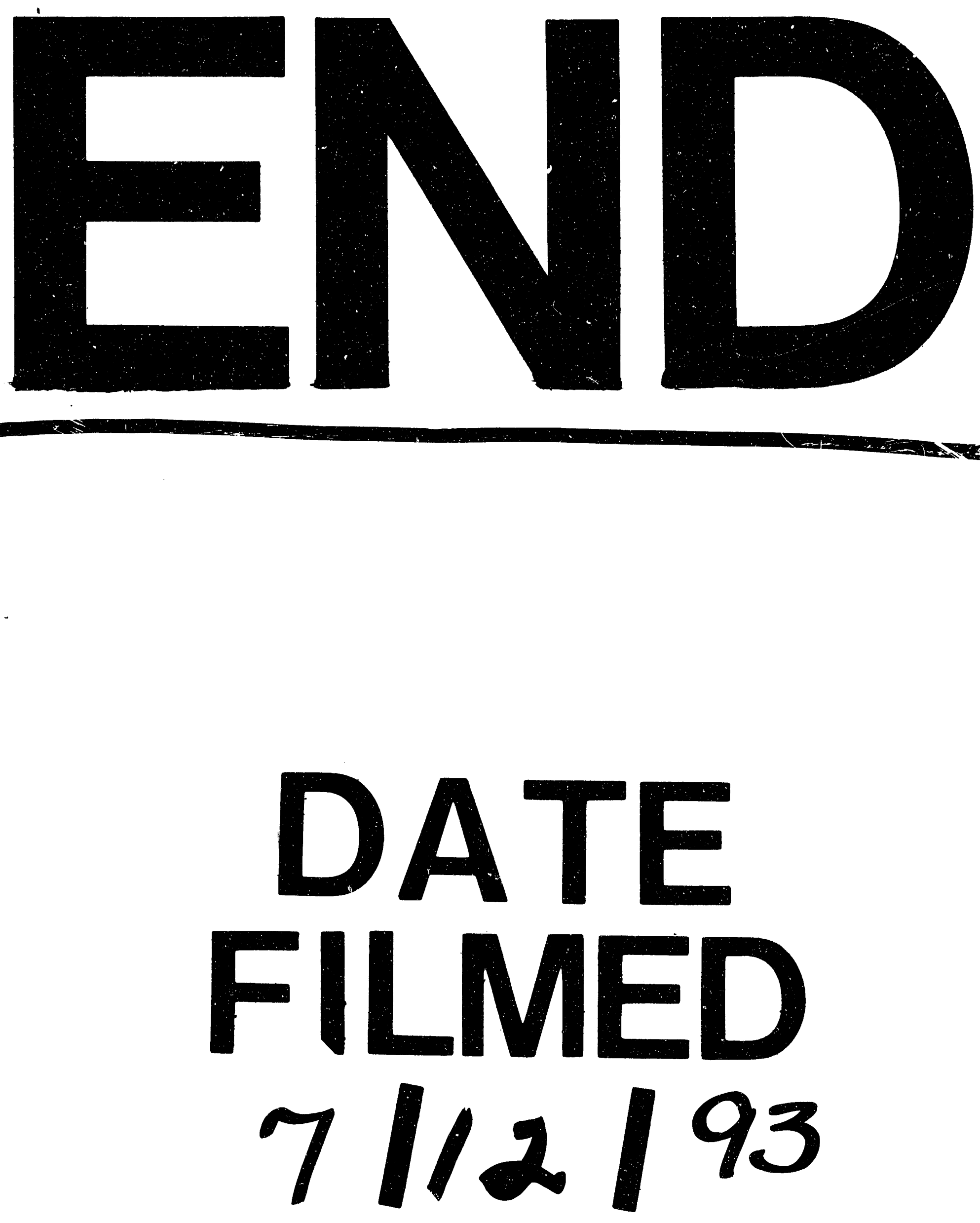